\title{
In vivo optical coherence tomographic imaging to monitor gingival recovery and the adhesive interface in aesthetic oral rehabilitation: A case report
}

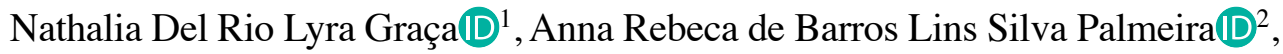 \\ Luana Osório Fernandes $\mathbb{D}^{1}$, Marlus da Silva Pedrosa $\mathbb{D}^{3}$, Renata Pedrosa Guimarães $\mathbb{D}^{1}$, \\ Saulo Cabral dos Santos $\mathbb{D}^{1}$, Anderson Stevens Leonidas Gomes $\mathbb{D}^{4}$, Claudio Heliomar Vicente da Silva $\mathbb{D}^{1, *}$ \\ ${ }^{1}$ Department of Prosthodontics and Bucco-Facial Surgery, School of Dentistry, Federal University of Pernambuco, Recife, Brazil \\ ${ }^{2}$ Department of Dentistry, UNINASSAU, Caruaru, Brazil \\ ${ }^{3}$ Department of Biomaterials and Oral Biology, School of Dentistry, University of São Paulo, São Paulo, Brazil \\ ${ }^{4}$ Department of Physics, Federal University of Pernambuco - UFPE, Recife, Brazil
}

\section{ABSTRACT}

The available methods for veneer evaluation are limited to clinical and radiographic examinations, which may not allow the appropriate identification of failure. In this report, we demonstrate the use of optical coherence tomography $(\mathrm{OCT})$ as a noninvasive diagnostic and follow-up method to evaluate gingival recovery and the adhesive interface in aesthetic oral rehabilitation involving periodontal plastic surgery and ceramic laminate veneers. OCT was efficient for evaluating both soft and hard tissues, as well as the quality of the adhesive interface. In conclusion, OCT was found to be a promising approach for the professional evaluation of aesthetic oral rehabilitation, as it was capable of generating images that enabled the analysis of gingival recovery and the adhesive interface. (Imaging Sci Dent 2019; 49: 171-6)

KEY WORDS: Esthetics, Dental; Gingivectomy; Dental Veneers; Dental Bonding; Tomography, Optical Coherence

An attractive smile increases social acceptance and improves the first impression that one makes. ${ }^{1}$ Periodontal plastic surgery and direct or indirect restorations, among others, are treatment approaches for oral aesthetic rehabilitation that may be indicated for patients concerned with the appearance of their smile. Adhesively cemented ceramic veneers transform the shape, color, and position of teeth, and provide aesthetic results with high treatment predictability. $^{2}$

The main challenge in the use of resin-based luting agents for cementation continues to be how to provide gap-free restorations. ${ }^{3}$ There is evidence of gap formation in the adhesive interface during and after polymerization. ${ }^{4}$ In light of such findings, the quality of the adhesive cementation is a predictor of the clinical success of dental restorations. ${ }^{5}$

Received November 11, 2018; Revised March 5, 2019; Accepted March 8, 2019 *Correspondence to: Prof. Claudio Heliomar Vicente da Silva

Department of Prosthodontics and Bucco-facial Surgery, Federal University of Pernambuco, Av. Prof. Moraes Rego, S/N, CDU. 50670-901 Recife/PE, Brazil

Tel) 55-81-2126-8344,E-mail) claudio_rec@ @otmail.com
Despite the high bonding capacity of resin cements, studies have been carried out to improve their properties, to monitor their clinical performance, and to identify and minimize or eliminate their failures.

In 1991, Huang et al. ${ }^{6}$ made an important contribution to biomedicine by proposing a new diagnostic method, optical coherence tomography (OCT). This technique enables the acquisition of cross-sectional images in high resolution and identification of the internal microstructure of materials and biological systems, with no need to cut the samples or expose patients to ionizing radiation.

OCT was initially proposed for ophthalmology. ${ }^{7}$ Among many other applications, this technique has been used in dermatology, ${ }^{8}$ oncology, ${ }^{9}$ and cardiology. ${ }^{10}$ It has also been widely employed in dental practice ${ }^{11}$ to evaluate the tooth-restoration interface, ${ }^{12}$ adhesive failures in indirect restorations, as well as fractures and cracks in composite resins, which could have positive implications for the noninvasive diagnosis of secondary caries. ${ }^{13}$

The follow-up of aesthetic oral rehabilitations is usually 

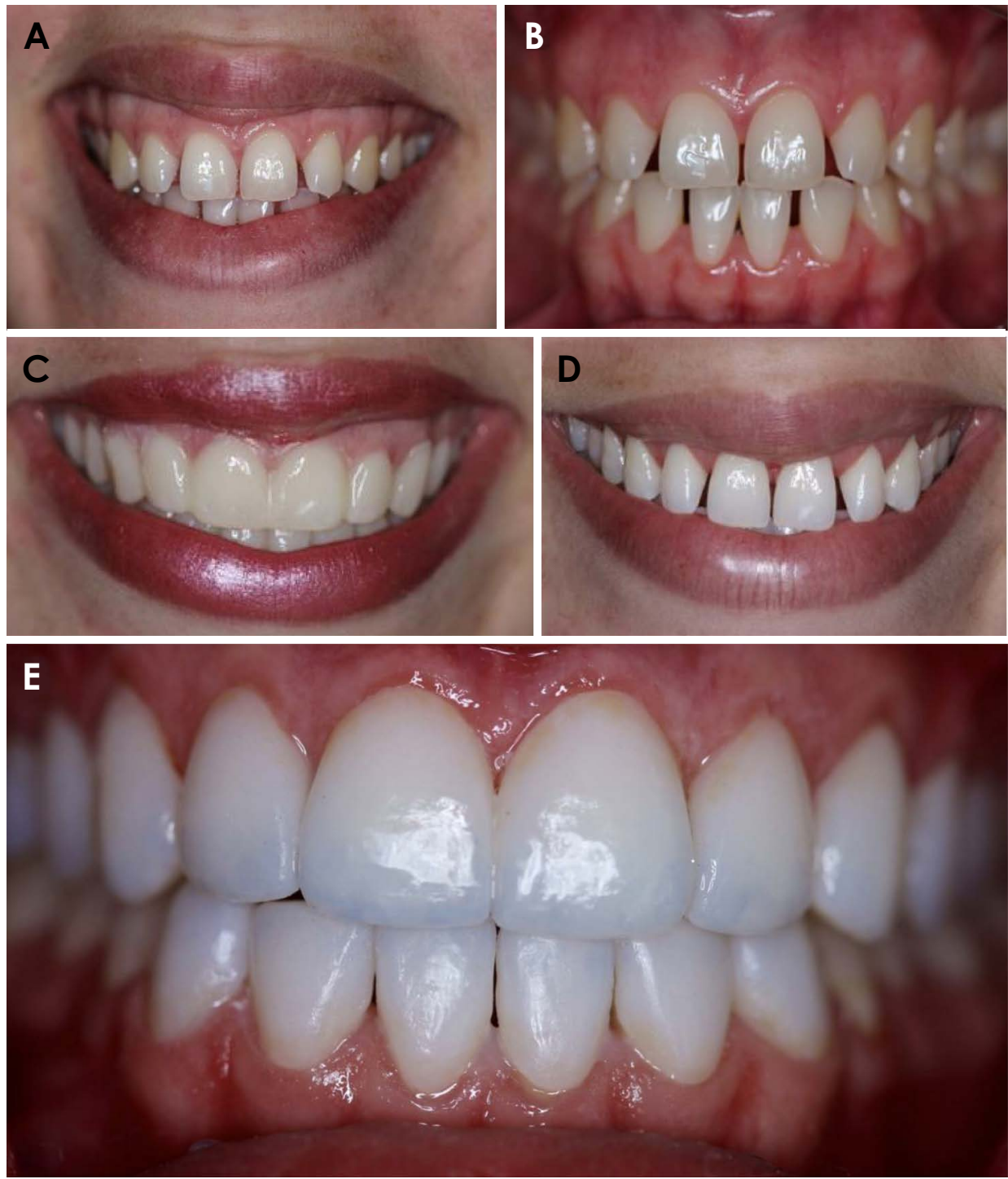

Fig. 1. Clinical photographs of the patient. Extraoral (A) and intraoral (B) photographs show the presence of yellowish teeth, generalized anterior diastema, and a gummy smile. C. Mock-up placed on teeth for the clinical simulation of the treatment outcome. The treatment consisted of periodontal plastic surgery, tooth whitening, and cementation of ceramic veneers. D. View of the patient's smile after periodontal plastic surgery and tooth whitening. E. Immediate clinical view after the cementation of the veneers. performed with visual inspection and radiographic examinations, which may hinder the evaluation of treatment success. ${ }^{14}$ This report describes the use of OCT as a noninvasive diagnostic and follow-up method for evaluating gingival recovery and the adhesive interface of ceramic laminate veneers.

\section{Case Report}

A 23-year-old woman reported dissatisfaction with the visual appearance of her smile due to the presence of yellowish teeth, generalized anterior diastema, and a gummy smile (Figs. 1A and B). Based on a clinical and radiographic examination, the following treatment plan was proposed: periodontal plastic surgery, tooth whitening, and cementation of ceramic veneers on the upper and lower arches.
The patient signed an informed consent form, and routine preoperative tests (blood count, coagulation, and fasting glucose) were requested. No abnormalities were found.

A 1325-nm Swept-Source OCT device (Thorlabs, Newton, NJ, USA) was used to image the front teeth. For the OCT readings, the patient remained seated with her head stabilized and perpendicular to the laser light source. The positions of the chin and glabella were standardized for all imaging sessions. Sagittal images of the cervical (Fig. 2A), middle (Fig. 2B), and incisal thirds (Fig. 2C) of the right maxillary central incisor were obtained through OCT before the treatment. It was possible to observe the enamel, dentin, dentin-enamel junction, gingiva, gingival sulcus, and incisal border.

An intraoral mock-up with A1 bis-acryl resin (Protemp 4; 3M, Maplewood, MN, USA) was constructed (Fig. 1C). 

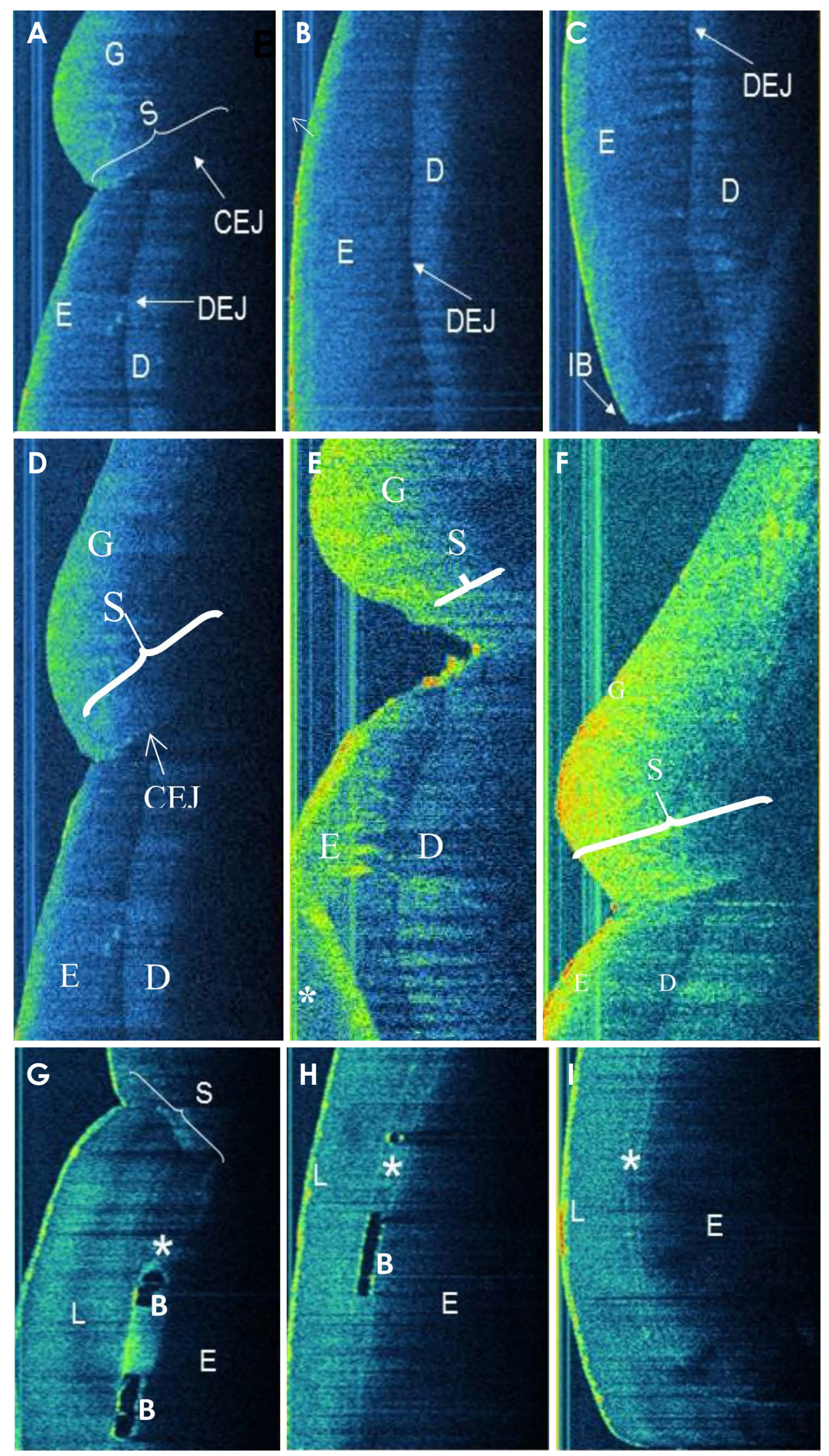

Fig. 2. Sagittal optical coherence tomography images. The cervical (A), middle (B), and incisal (C) thirds of the right maxillary central incisor showing a normal tooth and its surrounding periodontal structures, such as enamel (E), dentin (D), the dentino-enamel junction (DEJ), the cemento-enamel junction (CEJ), gingiva $(\mathrm{G})$, gingival sulcus $(\mathrm{S})$, and incisal border (IB). D. Cervical third of the tooth at 15 days (E) and 60 days (F) after the periodontal plastic surgery demonstrating gingival recovery, given the formation of a new gingival sulcus around the teeth and close contact of the gingiva with the tooth, as shown before surgery. The cervical $(\mathrm{G})$, middle $(\mathrm{H})$, and incisal (I) thirds of the right maxillary central incisor after cementation of the ceramic veneers showing the tooth and its surrounding periodontal structures, as well as the presence of the cementation line $(*)$, bubbles $(\mathrm{B})$, and the laminate veneer $(\mathrm{L})$. 
Periodontal plastic surgery was performed with the aid of a surgical guide. The bands of the keratinized soft tissue around the teeth were removed to obtain a parabolic shape. A flap was raised, exposing the local bone tissue. Osteotomy and osteoplasty were performed, aiming to restore the biological width. The flap was repositioned and sutured.

Gingival health was evaluated clinically and through OCT after 15 days and 2 months of tissue healing. The final sagittal OCT image of the cervical third of the right maxillary central incisor, when compared with the initial image and the image taken at 15 days, demonstrated gingival recovery, as shown by the formation of a new gingival sulcus around the teeth (Figs. 2D-F). Clinically, gingival health was observed. In addition, close contact of the gingiva with the tooth, as present before surgery, was shown in the OCT images. The gingiva was in the process of healing and reestablishment of its position around the tooth 15 days after the periodontal surgical procedure. The periodontal tissue showed normal patterns of healing given the formation of junctional epithelium (15 days) and maturation of connective tissue (45-60 days after periodontal surgery).

The patient was given the necessary material for at-home vital bleaching and was instructed to use a $16 \%$ carbamide peroxide whitening agent in a tray for 4 hours once a day for 2 weeks.

After the bleaching treatment was completed (Fig. 1D), minimal tooth preparations for ceramic veneers were carried out with diamond burs (KG Sorensen, Cotia, São Paulo, Brazil) and Sof-lex ${ }^{\mathrm{TM}}$ polishing discs (3M, Maplewood, MN, USA). Gingival retraction was performed to allow access for the impression material to record the finishing line of both arches. The impression material used was addition silicone (Express; 3M, Maplewood, MN, USA). Face bow and bite records were also taken.

The impressions were cast in type IV plaster and the physical models were mounted on a semi-adjustable articulator (A7 Plus; Bio-Art Soluções Inteligentes, São Carlos, SP, Brazil) and IPS e.max (Ivoclar Vivadent AG, Schaan, Liechtenstein) ceramic veneers were made.

The fit and the gingival adaptation of the veneers in the mouth were checked and the color shade of the cement was selected using Variolink Veneer Try-in pastes (Ivoclar Vivadent AG, Schaan, Liechtenstein).

The ceramic veneers were etched with $5 \%$ hydrofluoric acid (IPS Ceramic Etching Gel, Ivoclar Vivadent AG, Schaan, Liechtenstein) for 20 seconds, washed, and dried. The restorations were silanized (Monobond Plus, Vivadent AG, Schaan, Liechtenstein) for 1 minute. The adhesive (ExciTE F, Vivadent AG, Schaan, Liechtenstein) was applied to the internal face of each veneer and polymerized for 15 seconds (Radii-call LED curing light, SDI, Australia).

The teeth were etched with 37\% phosphoric acid (Total Etch, Vivadent AG, Schaan, Liechtenstein) for 15 seconds, washed and dried. The Adhesive (ExciTE F, Vivadent AG, Schaan, Liechtenstein) was applied to the dental enamel and polymerized for 15 seconds (Radii-call LED curing light; SDI, Bayswater, Australia).

The ceramic veneers were cemented with photopolymerizable resin cement (Variolink Veneer; Vivadent AG, Schaan, Liechtenstein) one by one, starting with the central teeth (Fig. 1E). The veneers were placed on the teeth under slight pressure followed by the removal of excess resin cement and polymerization for 30 seconds (Radii-call LED curing light, SDI, Bayswater, Australia). For the removal of remaining excess cement, scalpel blades, finishing and polishing discs, and abrasive polyester strips were used.

The sagittal OCT images after veneer cementation showed gingival health (Fig. 2G) and micrometer-sized bubbles in the cementation line (Figs. $2 \mathrm{H}$ and I). Given that the treatment was considered satisfactory, the patient was instructed about 6-month recall dental appointments to assess the health of the hard and soft tissues and the integrity of the veneers.

\section{Discussion}

OCT is a non-invasive and non-radioactive technique that is widely used in several clinical applications. In dentistry, OCT has been used for in vitro and in vivo evaluations of soft and hard tissues. ${ }^{15}$

Traditional diagnostic methods in dentistry are based on clinical, radiographic, and periodontal examinations, as well as probing. Radiographic images are 2-dimensional projections of 3-dimensional structures and expose the patient to radiation. Periodontal probing presents poor sensitivity and reliability. ${ }^{16}$ OCT, however, has been established as a comfortable, safe, and conservative diagnostic method.

OCT is able to detect qualitative and quantitative morphological changes of dental hard and soft tissues. Thus, it is an interesting method for evaluating aesthetic dentistry treatments, such as the one presented in this clinical case. The initial OCT images showed details from the hard and soft tissues, in which an area of normal gingiva with direct contact between the tissue and the tooth was observed. At 15 days after periodontal plastic surgery, the OCT images showed the healing process of the gingiva. At 60 days, gingival recovery and new gingival sulcus were observed, indicating that the conditions were ideal for continuing the 
aesthetic treatment.

Periodontal plastic surgery combined with restorative procedures, when indicated, can yield excellent aesthetic results. As the patient was searching for a harmonious and aesthetic smile, after surgery and tooth whitening, a treatment with ceramic veneers was proposed. Treatment with veneers is able to create naturally aesthetic results that involve morphological modifications related to tooth color, shape, contour, size, and volume, and it is well-accepted by periodontal tissues. ${ }^{17}$

Advances in adhesive technology have increased the use of ceramics. Initially, conventional feldspathic ceramics were used. However, those systems presented high failure rates and friability and low flexural strength, as well as resulting in significantly greater wear on the opposing teeth. ${ }^{18}$ New ceramic systems (leucite- and lithium disilicate-reinforced) were introduced, which yield excellent aesthetic results and better mechanical performance. ${ }^{19}$ The IPS e.max system, a lithium disilicate glass-ceramic, was selected in the case described herein given the possibility of working with a conservative adhesive approach. ${ }^{17}$

The thinness of the veneers means that the adhesive cement has a decisive effect on the final aesthetic result, especially when the ceramics have high translucency. The use of Try-in pastes allows selection of the color shade, making the final result predictable. ${ }^{20}$

The use of photopolymerizable resin cements is preferable for veneer cementation. Self- and dual-cured resin cements are more likely to show color changes over time. ${ }^{21}$ Color stability is also related to moisture contamination or insufficient photoactivation. ${ }^{22}$ For the clinical case presented herein, a photoactivated resin cement was used because it enabled easy removal of excess cement, different values and translucencies, and appropriate bonding and mechanical resistance.

The adhesive interface is a key element of successful treatment with ceramic veneers. The continuity between the tooth substrate, cement, and the internal face of a ceramic veneer is responsible for its fracture resistance and the solubility of cement in the oral environment. ${ }^{23}$ Ceramic veneers are extremely resistant when cemented on teeth. Air bubbles, however, may be incorporated during the cementation.

The adhesive cementation of 20 ceramic veneers was evaluated using OCT images. ${ }^{24}$ Adhesive failures such as bubbles and gaps in the cementation line were observed. Failures may have occurred due to the lack of uniformity and thickness of the cement. The air pockets seen may increase over time and culminate in detachment or fracture of the restoration. A low cementation pressure may also be a contributing factor to air bubble formation.

The available methods for veneer evaluation are limited to clinical and radiographic examinations, which may not allow the appropriate identification of failure. ${ }^{14,24}$ OCT has emerged as a high-resolution imaging modality that can be used without exposing patients to ionizing radiation. ${ }^{25}$ In this case report, OCT was chosen as an alternative to traditional clinical and radiographic examinations to evaluate indirect restorations due to its non-destructive and non-invasive characteristics.

OCT was found to be an efficient and promising approach for the professional evaluation of gingival recovery and the adhesive interface during aesthetic oral rehabilitation involving periodontal plastic surgery and ceramic laminate veneers. This approach could be used to investigate and monitor in vivo adhesive failure in ceramic laminate veneers.

\section{References}

1. Van der Geld P, Oosterveld P, Van Heck G, Kuijpers-Jagtman AM. Smile attractiveness. Self-perception and influence on personality. Angle Orthod 2007; 77: 759-65.

2. Morita RK, Hayashida MF, Pupo YM, Berger G, Reggiani RD, Betiol EA. Minimally invasive laminate veneers: clinical aspects in treatment planning and cementation procedures. Case Rep Dent 2016; 2016: 1839793.

3. Garcia-Godoy F, Krämer N, Feilzer AJ, Frankenberger R. Longterm degradation of enamel and dentin bonds: 6-year results in vitro vs. in vivo. Dent Mater 2010; 26: 1113-8.

4. Hayashi J, Shimada Y, Tagami J, Sumi Y, Sadr A. Real-time imaging of gap progress during and after composite polymerization. J Dent Res 2017; 96: 992-8.

5. Campos F, Valandro LF, Feitosa SA, Kleverlaan CJ, Feilzer AJ, de Jager N, et al. Adhesive cementation promotes higher fatigue resistance to zirconia crowns. Oper Dent 2017; 42: 215-24.

6. Huang D, Swanson EA, Lin CP, Schuman JS, Stinson WG, Chang W, et al. Optical coherence tomography. Science 1991; 254: 1178-81.

7. Al-Mujaini A, Wali UK, Azeem S. Optical coherence tomography: clinical applications in medical practice. Oman Med J 2013; 28: 86-91.

8. Moraes Pinto Blumetti TC, Cohen MP, Gomes EE, Petaccia de Macedo M, Ferreira de Souza Begnami MD, Tavares Guerreiro Fregnani JH, et al. Optical coherence tomography (OCT) features of nevi and melanomas and their association with intraepidermal or dermal involvement: a pilot study. J Am Acad Dermatol 2015; 73: 315-7.

9. John R, Adie SG, Chaney EJ, Marjanovic M, Tangella KV, Boppart SA. Three-dimensional optical coherence tomography for optical biopsy of lymph nodes and assessment of metastatic disease. Ann Surg Oncol 2013; 20: 3685-93.

10. Lopez AL 3rd, Wang S, Larin KV, Overbeek PA, Larina IV. Live four-dimensional optical coherence tomography reveals 
embryonic cardiac phenotype in mouse mutant. J Biomed Opt 2015; 20: 090501.

11. Hsieh YS, Ho YC, Lee SY, Chuang CC, Tsai JC, Lin KF, et al. Dental optical coherence tomography. Sensors (Basel) 2013; 13: 8928-49.

12. de Melo LS, de Araujo RE, Freitas AZ, Zezell D, Vieira ND, Girkin J, et al. Evaluation of enamel dental restoration interface by optical coherence tomography. J Biomed Opt 2005; 10: 064027.

13. Braz AK, Kyotoku BB, Braz R, Gomes AS. Evaluation of crack propagation in dental composites by optical coherence tomography. Dent Mater 2009; 25: 74-9.

14. Alhekeir DF, Al-Sarhan RA, Al Mashaan AF. Porcelain laminate veneers: clinical survey for evaluation of failure. Saudi Dent J 2014; 26: 63-7.

15. Machoy M, Seeliger J, Szyszka-Sommerfeld L, Koprowski R, Gedrange T, Woźniak K. The use of optical coherence tomography in dental diagnostics: a state-of-the-art review. J Healthc Eng 2017; 2017: 7560645.

16. Xiang X, Sowa MG, Iacopino AM, Maev RG, Hewko MD, Man A, et al. An update on novel non-invasive approaches for periodontal diagnosis. J Periodontol 2010; 81: 186-98.

17. Reddy MS. Achieving gingival esthetics. J Am Dent Assoc 2003; 134: 295-304.

18. Vanlıŏ̆lu BA, Kulak-Özkan Y. Minimally invasive veneers: cu- rrent state of the art. Clin Cosmet Investig Dent 2014; 6: 101-7.

19. Morimoto S, Albanesi RB, Sesma N, Agra CM, Braga MM. Main clinical outcomes of feldspathic porcelain and glass-ceramic laminate veneers: a systematic review and meta-analysis of survival and complication rates. Int J Prosthodont 2016; 29: $38-49$.

20. Silva LH, Lima E, Miranda RB, Favero SS, Lohbauer U, Cesar PF. Dental ceramics: a review of new materials and processing methods. Braz Oral Res 2017; 31 (suppl 1): e58.

21. Chadwick RG, McCabe JF, Carrick TE. Rheological properties of veneer trial pastes relevant to clinical success. Br Dent $\mathbf{J}$ 2008; 204: E11.

22. Rodrigues RB, Lima E, Roscoe MG, Soares CJ, Cesar PF, Novais VR. Influence of resin cements on color stability of different ceramic systems. Braz Dent J 2017; 28: 191-5.

23. Pissaia JF, Correr GM, Gonzaga CC, da Cunha LF. Influence of shade, curing mode, and aging on the color stability of resin cements. Braz J Oral Sci 2015; 14: 272-5.

24. de Andrade Borges E, Cassimiro-Silva PF, Fernandes LO, Gomes AS. Study of lumineers' interfaces by means of optical coherence tomography. Proc. SPIE Biophotonics South America 2015; 9531: 953147.

25. Hall A, Girkin JM. A review of potential new diagnostic modalities for caries lesions. J Dent Res 2004; 83: C89-94. 